\title{
La perspectiva de profesores de idiomas en formación sobre los rasgos de un buen profesional. Estudio de caso
}

The perspective of undergraduate language teachers about the traits of a
good professional. Case study

A perspectiva de professores de línguas em formação sobre as características de um bom profissional. Estudo de caso

Laura Emilia Fierro López Universidad Autónoma de Baja California, Facultad de Idiomas, México

laurafierro@uabc.edu.mx http://orcid.org/0000-0002-8418-4905

Rey David Román Gálvez Universidad Autónoma de Baja California, Facultad de Ciencias Humanas, México divad@uabc.edu.mx http://orcid.org/0000-0001-9703-7915

Lilia Martínez Lobatos Universidad Autónoma de Baja California, Facultad de Idiomas, México liliam@uabc.edu.mx http://orcid.org/0000-0001-9423-1384

\section{Resumen}

El siguiente es un estudio transeccional descriptivo diseñado con el objetivo de identificar los rasgos de un buen profesional desde la perspectiva de estudiantes en proceso formativo para la docencia de idiomas de una universidad pública en México. La muestra se conformó por 322 estudiantes de cuatro unidades académicas, lo que representa $50.95 \%$ de la población. Los participantes, mediante una escala ipsativa de 16 rasgos, categorizaron los más importantes. Se identificó que los estudiantes valoran, primordialmente, el conocimiento, la formación, la preparación y la competencia profesional, así como la ética 


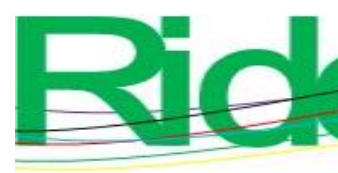

Revista Iberoamericana para la
Investigación y el Desarrollo Educativo
ISSN $2007-7467$

profesional. El rasgo que identificaron como menos importante fue la capacidad emocional. Se concluye el valor que los estudiantes atribuyen a las competencias cognitivas y técnicas, aunque no alcanzan a valorar rasgos relacionados con las competencias sociales y afectivoemocionales. Los resultados permiten señalar la relevancia de fortalecer rasgos de las competencias sociales y afectivo-emocionales, además de otros de las competencias éticas, en el trayecto formativo de los estudiantes. Ello de una forma reflexiva para que los estudiantes puedan analizar y consolidar aspectos que les permitan una formación humanística y de responsabilidad social para impactar de forma favorable en su desempeño profesional.

Palabras clave: enseñanza de idiomas, ética profesional, formación de profesores, formación profesional, identidad profesional.

\begin{abstract}
This paper presents a descriptive transectional study with the purpose of identifying the most significant traits of being a good professional, from the opinion of the bachelor in language teaching students of a public university in Mexico. A sample of 322 students from four academic campus represented a 50.95 percentage of the population where, by means of an ipsative scale of 16 traits, they categorized the most important. It was identified that the most valued traits are knowledge, education, academic preparation, and professional competence, as well as professional ethics. The least valued trait is emotional abilities. Findings show the high value students give to cognitive and technical competences, although, they fail to appraise traits related to social and affective-emotional competences. The results allow us to point out the relevance of strengthen traits of social and affective-emotional competences, in addition to other ethical competences, in the educational path of students. This in a reflective way, so that students can analyze and consolidate aspects that allow them a humanistic and social responsibility training that have a favorable impact on their professional performance. Keywords: teaching languages, professional ethics, teacher education, professional education, professional identity.
\end{abstract}




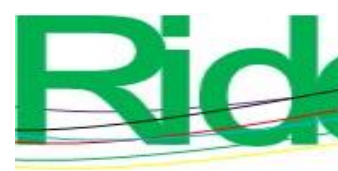

Revista Iberoamericana para la
Investigación y el Desarrollo Educativo ISSN $2007-7467$

\section{Resumo}

A seguir, um estudo transversal descritivo elaborado com o objetivo de identificar as características de um bom profissional a partir da perspectiva de alunos em processo de formação para o ensino de línguas em uma universidade pública mexicana. A amostra foi composta por 322 alunos de quatro unidades acadêmicas, o que representa 50,95\% da população. Os participantes, usando uma escala ipsativa de 16 características, categorizaram as mais importantes. Identificou-se que os alunos valorizam, prioritariamente, o conhecimento, a formação, a preparação e a competência profissional, bem como a ética profissional. A característica que identificaram como menos importante foi a capacidade emocional. Conclui-se o valor que os alunos atribuem às competências cognitivas e técnicas, embora não consigam avaliar traços relacionados às competências sociais e afetivoemocionais. Os resultados permitem apontar a relevância do fortalecimento de traços de competências sociais e afetivo-emocionais, bem como de outras competências éticas, no percurso educacional dos alunos. Isso é feito de forma reflexiva para que os alunos possam analisar e consolidar aspectos que lhes permitam uma formação humanística e de responsabilidade social ter um impacto favorável em seu desempenho profissional.

Palavras-chave: ensino de línguas, ética profissional, formação de professores, formação profissional, identidade profissional.

Fecha Recepción: Julio 2020

Fecha Aceptación: Enero 2021

\section{Introducción}

La formación de profesionales en la enseñanza de idiomas en México, a nivel de licenciatura, cuenta con una génesis reciente en comparación con otras carreras universitarias que ya poseen el reconocimiento social. La oferta educativa inició a partir de 1980, y con mayor auge en los años noventa, con la posterior creación de posgrados en el área de lenguas. Lo novel de este campo, el endeble reconocimiento social de un perfil profesional para la enseñanza de lenguas, así como la alta demanda en esta área conducen a determinar las razones para la inserción de profesores que carecen de formación profesional para la enseñanza en los diversos espacios educativos mexicanos. Por ello, persisten enormes retos, limitaciones y vacíos en la disciplina de enseñanza de lenguas. 


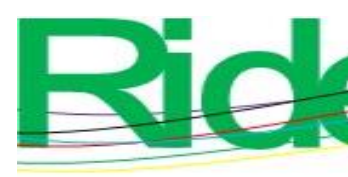

Revista Iberoamericana para la
Investigación y el Desarrollo Educativo
ISSN $2007-7467$

Se destaca el papel que desempeña el profesor en el aprendizaje de lenguas, por tanto, la formación lingüística y pedagógica tienen impacto para consolidarlo. Esto constituye la base para sustentar la enseñanza. Asimismo, se acentúa la relevancia de la formación ética y de valores en los profesores de lenguas. En los diseños curriculares de programas educativos de instituciones de educación superior, basados en competencias, se agrupan elementos que contribuyen a la formación integral del futuro profesional, mediante el desarrollo de conocimientos, habilidades y actitudes. El propósito es forjar ciudadanos con capacidades más allá de la adquisición de conocimientos y promover formas de relacionarse que permitan una sociedad más justa e inclusiva.

La finalidad de la formación integral en ocasiones puede ser poco perceptible para los estudiantes, ya que la obtención de "buenas" calificaciones parece ser su principal preocupación, lo cual se sigue valorando como el dominio del contenido teórico de las diversas asignaturas del programa. Esto va en desacuerdo con la formación basada en competencias, donde también deben incorporarse las habilidades y actitudes. Aunque la dimensión actitudinal se incorpora en el diseño curricular de estos programas educativos de formación, de forma vertical y transversal, es un tema poco claro o sobresaliente para los estudiantes. Por eso, es necesario explorar cómo lo perciben los alumnos.

La ética y los valores han sido estudiados por las ciencias educativas y la filosofía; sin embargo, no existen resultados de investigaciones en esta área de formación que permitan analizar los avances y realizar iniciativas pertinentes para hacer aportaciones en este tipo de programas curriculares.

A partir de los planteamientos anteriores, se presenta un informe de investigación donde se aborda la opinión de profesores de idiomas en formación sobre los rasgos de un buen profesional. En estos rasgos se agrupan características de acuerdo con las dimensiones propuestas por Hirsch (2005): competencias cognitivas y técnicas, sociales, éticas y afectivoemocionales.

\section{Formación profesional de profesores de lenguas}

En México, existe una demanda creciente de profesores de lenguas extranjeras originada, primordialmente, por la situación que prevalece en las instituciones de educación superior respecto a la "perspectiva de la internacionalización del currículo, apuntaladas por la Unesco y la OCDE” (Asociación Nacional de Universidades e Instituciones de Educación 


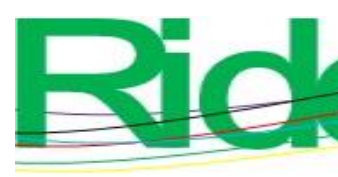

\section{Revista Iberoamericana para la Investigación y el Desarrollo Educativo ISSN 2007 - 7467}

Superior [Anuies], 2019, p. 5). Esto se refleja en las cifras ascendentes de estudiantes de idiomas en América Latina y el Caribe (Anuies, 2019).

De manera reciente, la necesidad de profesores de lenguas extranjeras es también perceptible en otros niveles educativos, por ejemplo, con los cambios en el currículo de educación básica en el año 2009, al incorporar una asignatura de inglés en los niveles preescolar y primaria públicas (Secretaría de Educación Pública [SEP], 2011) y la Estrategia Nacional de Inglés derivada del Nuevo Modelo Educativo (SEP, 2017).

Además, la enseñanza de idiomas es parte del fortalecimiento lingüístico de lenguas originarias, las cuales en su mayoría se encuentran en riesgo. Por ello, dentro de las estrategias de planificación lingüística para su revitalización, conservación y promoción es parte relevante el perfil y la formación de docentes para atender estas necesidades.

Estas y otras transformaciones ponen en discusión tres aspectos fundamentales para la conformación del perfil profesional del docente de lenguas: 1) conocimientos teóricos y metodológicos para la enseñanza, 2) habilidades para generar ambientes de aprendizaje, y 3) competencia comunicativa en el idioma de instrucción, elementos indispensables para hacer frente a la enseñanza de lenguas. Queda de manifiesto el hecho de requerir profesores que cuenten con las habilidades profesionales para este efecto.

Paquay et al. (2005) enuncian como "habilidades profesionales" a todo el conjunto de conocimientos, esquemas de acción y actitudes que se demandan en el ejercicio de la profesión docente. Facultar a los profesores en este conjunto de habilidades profesionales resulta un proceso complejo, ya que se debe esperar que los profesores tengan como base conocimientos acerca de teorías, métodos de enseñanza y contenidos de la disciplina para el manejo de estas habilidades profesionales (Fierro, Martínez y Cordero, 2014, p. 4).

Sin embargo, los procesos y cambios que se viven en la sociedad actual ponen en énfasis, además de una formación profesional en el área de lenguas, en la incorporación en el sistema educativo de profesores con una "formación en valores de forma responsable y comprometida con la sociedad en general y con las nuevas generaciones de alumnos en particular” (Beltrán, Torres, Beltrán y García, 2005, p. 399).

Los conocimientos profesionales son los que integran el esquema de análisis de las situaciones y, por ende, determinan sus acciones. Son los que median en la planificación y evaluación del profesor para la toma de decisiones de las acciones a realizar. Asimismo, está delimitado por las actitudes — como establecen Paquay, Altet, Charlier y Perrenoud (2005)— 


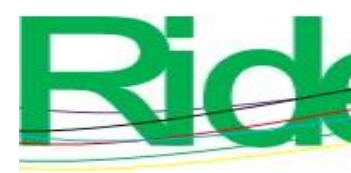

Revista Iberoamericana para la
Investigación y el Desarrollo Educativo
ISSN $2007-7467$

en la convicción que tienen por la educación de sus estudiantes, el respeto hacia el prójimo, la conciencia de las propias representaciones, la apertura a la colaboración y el compromiso profesional, destacando el aspecto pedagógico-social en el aprendizaje de los estudiantes.

En el área de la enseñanza de lenguas ha tenido gran significancia indagar acerca de cómo los conocimientos teóricos y las creencias de los profesores guían las acciones efectuadas en el aula (Breen, 1991; Freeman, 1989; Richards, 1998, 2011; Woods, 1996). Por eso, Freeman (1989) señala la necesidad de investigar acerca de este proceso. Este autor afirma que la formación en esta área se encontraba fragmentada y sin una base conceptual sólida que rigiera los esfuerzos realizados para desarrollar programas de formación docente, y se aducía que eran mayormente influenciados por distintas disciplinas.

La afirmación de Freeman cobra sentido al considerar que el profesor de lenguas debe contar con conocimientos y habilidades de enseñanza, del mismo modo que un profesor de otro campo de instrucción. Como menciona Richards (1998), el profesor debe dominar las competencias genéricas de la pedagogía y nociones de otras disciplinas, como la lingüística, psicolingüística y sociolingüística, articuladas de manera coherente en su formación.

Posteriormente, Richards (2011) amplía el dominio de contenidos al exponer los conocimientos, creencias y habilidades para el profesional en la enseñanza de lenguas. En este, identifica dimensiones esenciales que permiten establecer un marco para conceptualizar la naturaleza de competencia, pericia y profesionalismo. Entre ellas se circunscriben, además de lo propuesto con anterioridad por este autor (Richards,1998), el conocimiento del contexto que atiende la perspectiva sociocultural donde se sitúa la enseñanza. En él se encuentran aspectos como los propósitos particulares de la escuela, el currículo que se implementa, la cultura escolar y las características de sus profesores y estudiantes.

Este conocimiento contextual, incluido dentro de la sociolingüística, permite al profesor reconocer la manera de actuar ante determinadas situaciones, después de entender las normas de práctica, valores y patrones de participación de la escuela. El profesor forma su actuar en un proceso de socialización que se logra en el contexto escolar como miembro de una comunidad de práctica.

Se toma el caso de la Unión Europea que valora la lengua como un sistema de signos en un entorno social, donde se enfatiza en el conocimiento de las características del contexto. Por la cercanía de los países, existe un mayor acceso a la movilidad y a la empleabilidad más allá de las fronteras. Por ello, le apuestan a la calidad en la formación de profesores de lenguas para facilitar estos procesos. 


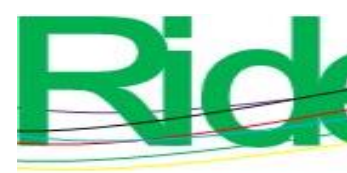

Revista Iberoamericana para la Investigación y el Desarrollo Educativo ISSN 2007 - 7467

En el documento European profile of language teacher education - a frame of reference (Kelly, Grenfell, Allan, Kriza y McEvoy, 2004), se establece un marco de referencia que contiene información acerca de contenidos, ejemplos de buenas prácticas e innovaciones que pueden ser considerados en programas de formación. Constituye una base, a manera de recapitulación de principios o lineamientos, enfocada en la estructura de cursos educativos, contenidos (conocimientos, estrategias y habilidades) y valores para este tipo de programas formativos.

Aunque en el manuscrito europeo se aclara que está dirigido a examinar la formación de profesores de lenguas extranjeras - no para profesores que enseñen su lengua materna-, sí es una guía para el desarrollo de programas en este campo. En este es parte medular, al igual que en otros desarrollos curriculares, que los profesores identifiquen y comprendan la naturaleza de la enseñanza y el aprendizaje de lenguas para poner en práctica en situaciones reales, lo que es adquirido en su formación inicial, además del desarrollo profesional continuo como parte de la actualización y mejora en su profesión.

La reflexión en acción adquiere contribuciones importantes en esta búsqueda de la mejora continua del profesor. De acuerdo con Schön (1983), para construir el conocimiento profesional, se fundamenta en la experiencia y reflexión de las acciones realizadas en la práctica. La reflexión favorece el desarrollo profesional para la docencia, lo que impacta positivamente en el proceso educativo en particular y, en consecuencia, en la sociedad en conjunto (Fierro, 2019).

Sin lugar a dudas, la reflexión es un elemento importante para el desarrollo profesional. En esta introspección es donde se demanda que se incorporen elementos relacionados con las representaciones sobre su profesión, que incluyen la ética y los valores profesionales.

Los profesores de lenguas no están exentos en esta configuración; además, se precisa incorporar en su formación el bagaje sociocultural del trabajo en equipo, la colaboración y las redes que fomenten una verdadera "participación colectiva profesional” (Imbernón, 2001, p. 3).

La formación docente que se desarrolla en las instituciones de educación superior establece el inicio, mientras que el aprendizaje posterior se realiza en la escuela. Las propias metas, normas de conducta, valores y creencias compartidos se convierten en el currículo oculto, en ocasiones más poderoso que el currículo determinado por la institución. 


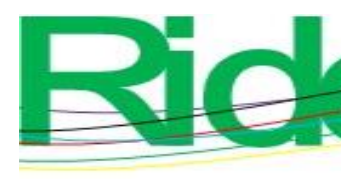

Revista Iberoamericana para la
Investigación y el Desarrollo Educativo
ISSN $2007-7467$

Es dentro de esta perspectiva sociocultural del contexto donde se desarrolla la dimensión de identidad del profesor de lenguas, que se manifiesta en los roles que el profesor desempeña, ya sean sociales o culturales en su entorno educativo.

\section{Identidad profesional, ética y valores}

La identidad profesional es una construcción que se materializa a partir de las experiencias personales, pero también se sustenta en los sucesos sociales vividos en la comunidad y en la manera que se le percibe en la sociedad, de ahí que se le llame un proceso de construcción individual y colectiva (Bolívar, 2007; Lopes, 2007; Richards, 2011).

Lo vivido en la colectividad determina, en buena parte, esta identidad; afecta la manera en que el profesor se desempeña en su campo y en el tipo de discurso que adopta, ya que mediante él se manifiesta el grupo al que pertenece, así como la representación que tiene del sentido de ser docente. Igualmente, en el papel que ejerce dentro del salón de clases, la escuela y la comunidad; incluso en lo que el profesor hace para influir en las decisiones que conciernen a su quehacer docente.

Desde estos preceptos de colectividad se construye la ética profesional y se establece en la cultura, siendo manifiesta en las normas o códigos de conducta observables en la profesión. Se reconoce la función social que desempeña la docencia en la sociedad, así como su impacto. Es lógico determinar dentro de sus responsabilidades principales el logro de los aprendizajes en los alumnos de los futuros profesores, para este propósito, dentro de sus deberes, es poseer el conocimiento de la disciplina que imparte y actualizarse en los contenidos que enseña (Hortal, 2000).

La interrogante que surge es si se puede desarrollar una identidad profesional desde las instituciones de educación superior. Desde la formación inicial, como una trayectoria profesional, se adquieren los puntos de referencia para formarse las representaciones individuales de cómo es el desempeño para que el profesor se justifique, analice y evalúe.

Sin embargo, esta identidad se enfrenta a la realidad del contexto actual, y aunque convergen, están en una continua adaptación por los contrastes que existen en lo adquirido durante su formación inicial. En ocasiones, se idealiza un concepto de identidad, y a partir de lo que experimentan en sus ambientes de trabajo, les hace desistir en seguir con esa identidad y crear una nueva (Lopes, 2007). 


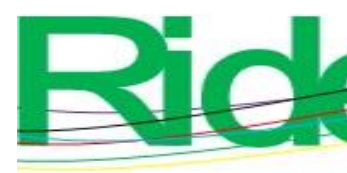

Revista Iberoamericana para la Investigación y el Desarrollo Educativo ISSN 2007 - 7467

Se argumenta que esta crisis de identidad se debe a que la formación inicial es académica más que profesional, que carece de bases sólidas para resistir a la destrucción de la identidad profesional. Es decir, se desarrollan más los aspectos de la enseñanza: teorías, metodologías, desarrollo de habilidades, el "argot" del medio, y menos la construcción de una identidad profesional profunda y sólida. Lopes (2007) lo atribuye a la política educativa de formación que fracasa desde su base en el desarrollo de una identidad.

En el campo de la enseñanza de lenguas en México, la identidad del profesor se encuentra fragmentada debido a diversos factores. Se puede aducir, primordialmente, a lo novel de los programas de profesionalización en el área de lenguas a nivel de licenciatura (Ramírez, 2007, 2010). También se puede atribuir a los vacíos en la inserción de profesores con formación en el área de la enseñanza de lenguas en los espacios educativos. Se otorga una alta valoración a la competencia comunicativa en la lengua de instrucción como el único factor a considerar para hacer frente a las necesidades en los diversos espacios educativos. Sin embargo, estas circunstancias vulneran la construcción de la identidad profesional, lo que —de acuerdo con Bolívar (2007), Lopes (2007) y Richards (2011) — afecta las representaciones que tienen los sujetos acerca del sentido de ser docente.

Otra de las partes a destacar, dentro de las dimensiones propuestas por Richards, es la concerniente a la participación del profesor en una comunidad de práctica. Es el sentirse miembro de un grupo de personas que comparten un fin común, que conciben a la educación desde una perspectiva filosófica, con un conjunto de saberes para orientar el conocimiento y enriquecer el actuar del ser humano, resolver las problemáticas y asuntos comunes.

La comunidad de práctica es parte de la identidad del profesor donde aprende a partir de las acciones colaborativas con otros colegas, ya sea para solucionar problemas, presentar propuestas, cambiar prácticas, planear cursos, evaluaciones, entre otras actividades. De esta manera, el profesor puede indagar acerca de lo que viven en sus aulas para buscar soluciones o explicaciones.

Las dimensiones citadas — según el planteamiento de Richards (2011) — ayudan a configurar el perfil profesional del docente de lenguas. Se requiere una formación teórica y práctica, y no solo hablar la lengua de instrucción para considerarse un profesional en la enseñanza de idiomas. Los congresos, las revistas especializadas, las organizaciones profesionales y, primordialmente, el trabajo realizado por universidades para desarrollar programas de formación a nivel de licenciatura y posgrado en el área de lenguas contribuyen con este propósito. 


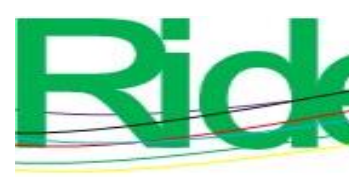

Revista Iberoamericana para la Investigación y el Desarrollo Educativo ISSN 2007 - 7467

En los proyectos educativos universitarios se establecen los conocimientos, habilidades y actitudes; en otras palabras, se destaca la formación integral de los futuros egresados. No obstante, aunque cada vez se avanza con mayor precisión en diseños curriculares que contribuyan a alcanzar estos logros, todavía subsisten dificultades para presentar modelos o enfoques para formar a profesionales con un sentido de la ética relacionado con el ejercicio de su profesión. Es decir, con cualidades o características personales que le orienten a actuar de acuerdo con normas en una dimensión ética-moral.

Cada vez es más visible en las profesiones — como lo asevera López Zavala (2013)— un endeble compromiso hacia la responsabilidad social, que reduce "la identidad profesional a su dimensión económica" (López Zavala, 2013, p. 15) debido a lo inestable y etéreo de los compromisos duraderos e interés en convicciones sociales, características a las que Bauman (2011) denomina sociedad líquida. López Zavala lo explica como una respuesta para enfrentar la vorágine de demandas de la empleabilidad, que simplifica la formación a su dimensión técnica.

La ética profesional, desde un sentido amplio, "se enfoca en las virtudes y los roles profesionales" (Luna-Serrano, Valle-Espinoza y Osuna-Lever, 2010, p. 4). Los aspectos deontológicos son inherentes a las profesiones, donde no quedan exentos los profesores de lenguas. La ética y los valores profesionales adquieren relevancia, primordialmente, derivados de los deberes y principios reconocidos socialmente dentro de lo que se denomina ser un buen maestro.

En este sentido, se puede decir que se han incrementado considerablemente los estudios que contribuyen al conocimiento sobre la ética profesional en México $-\mathrm{y}$ a nivel mundial_- Estos trabajos documentan los problemas y dilemas éticos que identifican los profesionales en su ejercicio. Sin embargo, es cada vez más frecuente y de mayor intensidad la conducta no ética en profesionistas, profesores, investigadores y estudiantes (Hirsch y Pérez-Castro, 2007), lo que sirve para cuestionar el papel de la formación universitaria en "la difusión de valores para crear un orden social más justo" y "la autoconciencia de los derechos humanos" (Luna-Serrano et al., 2010, p. 3).

Esta es un área significativa de estudio que se encuentra en una etapa incipiente con propuestas de formación en la dimensión valoral en instituciones de educación superior, así como contextualizadas en la ética de la profesión docente, particularmente relevante a partir de los cambios generados en el sistema educativo mexicano en los últimos tiempos (Hirsch y Pérez-Castro, 2007). En estudios realizados, donde los sujetos participantes son estudiantes 


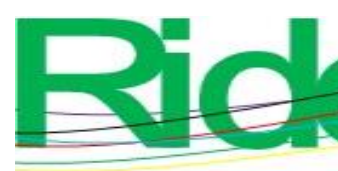

Revista Iberoamericana para la
Investigación y el Desarrollo Educativo
ISSN $2007-7467$

universitarios, destacan los valores como la responsabilidad, la honestidad y el conocimiento, mientras que se identifican como menos importantes el trabajo en equipo y la comunicación (Luna-Serrano et al., 2010). Los docentes, en cambio, valoran la integridad, el respeto y el profesionalismo, estableciendo un rango menor a las competencias sociales (Serna y Luna, 2011).

En este sentido, se destaca "la importancia de una adecuada formación ética del profesorado para su correcto desempeño profesional" (Sanz y Hirsch, 2016, p. 141). Se pone acento en el desarrollo no solo de las competencias teórico-pedagógicas para la enseñanza de lenguas o "el dominio de competencias para el buen desempeño en la dimensión técnica" (López Zavala, 2013, p. 16), sino también en la formación de profesionales con un alto compromiso social y ético para retribuir a la sociedad el bien recibido en su instrucción universitaria.

Este estudio, por tanto, se sitúa en un programa de licenciatura dirigido a la formación de profesores de lenguas. En su perfil de egreso se contempla que los estudiantes deben poseer conocimientos teóricos y metodológicos para la enseñanza de lenguas, habilidades para generar ambientes de aprendizaje, así como fortalecer la competencia comunicativa en inglés y desarrollar una tercera lengua meta. En este sentido, la formación lingüística y pedagógica impacta el desempeño en el ejercicio de la profesión, pero también se destaca la relevancia de la formación ética y valoral en los profesores de lenguas.

Dentro su trayecto formativo se encuentra la inserción de la asignatura Ética, Responsabilidad Social y Transparencia, con el propósito de contribuir a la reflexión sobre la importancia de los valores y la ética en el ejercicio de los futuros profesionales. La asignatura se incorpora en el tronco común como obligatoria para los estudiantes de los dos programas educativos de licenciatura que se ofertan en la facultad. En las etapas posteriores —disciplinaria y terminal — se contempla que la ética y los valores sean incorporados de manera transversal en cada una de las materias del plan de estudios.

En concordancia con el modelo educativo de la institución, la instrumentación de las iniciativas anteriores favorece la formación integral, que comprende no únicamente las competencias y habilidades para incorporarse al campo de trabajo, sino también los valores y actitudes necesarios para formar al futuro profesionista comprometido con su entorno (Universidad Autónoma de Baja California [UABC], 2014). Se pretende que mediante este modelo los estudiantes universitarios reflexionen sobre la ética y valores en el quehacer profesional, "una ética que intente orientar las conductas profesionales" para "establecer un 


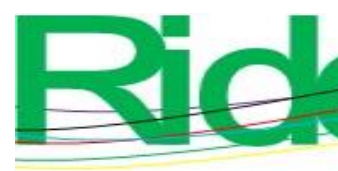

Revista Iberoamericana para la
Investigación y el Desarrollo Educativo
ISSN $2007-7467$

diálogo interdisciplinar con los saberes especializados en los que se basa el ejercicio de cada profesión" (Hortal, 2002, p. 15).

A partir de los planteamientos señalados, se establece el objetivo de identificar los rasgos más importantes de ser un buen profesional desde la perspectiva de los estudiantes de la licenciatura en Docencia de Idiomas. De este modo se conocerá la opinión de los estudiantes para analizar las tensiones e inconsistencias que puedan ser evidentes en la formación inicial de los sujetos respecto a las competencias propuestas por Hirsch (2005), donde se incluye la dimensión ética-valoral.

La función formadora de las instituciones educativas es evitar o modificar actitudes negativas como "el individualismo, la competitividad, la falta de vínculos con el mundo exterior, el razonamiento superficial, la mediocridad y la falta de preparación de los profesores en la educación moral” (Grass, 2001, citado por Beltrán et al., 2005, p. 399). La relevancia de identificar los valores profesionales y éticos en el proceso de formación de los profesores de lenguas se encuentra en que no se han hallado estudios sobre la ética y los valores contextualizados en esta área disciplinaria.

Por consiguiente, es necesario documentar la problemática estudiándola de forma sistemática, con una perspectiva integradora, donde a partir del análisis se contribuya a generar acciones que posibiliten mejorar las condiciones para alcanzar los objetivos propuestos y, con ello, fomentar una formación que contribuya al desarrollo de profesionales en el área de la enseñanza de lenguas con los valores propios de su profesión, así como para asumir su compromiso con la sociedad. De esta manera se pretende enriquecer la literatura sobre el tema en el contexto mexicano y en la disciplina de formación de docentes de lenguas para ofrecer un panorama sobre los valores presentes en los actuales estudiantes.

\section{Método}

Se realizó un estudio transeccional descriptivo con la participación de estudiantes de la licenciatura en Docencia de Idiomas de la Facultad de Idiomas de la Universidad Autónoma de Baja California (UABC), en cuatro municipios del estado de Baja California. 


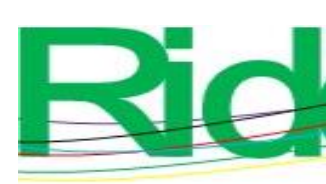

Revista Iberoamericana para la Investigación y el Desarrollo Educativo ISSN 2007 - 7467

\section{Instrumento de recolección de datos}

Se utilizó un instrumento con una escala ipsativa integrado por 16 rasgos de ser un buen profesional (tabla 1), los cuales fueron jerarquizados por los participantes según los más importantes. Además, se incorporó una sección para recolectar datos demográficos: sexo, edad, unidad académica, semestre y turno al que pertenecen.

Tabla 1. Rasgos de ser buen profesional

\begin{tabular}{|c|c|}
\hline Competencias & Rasgos \\
\hline $\begin{array}{l}\text { Competencias cognitivas y } \\
\text { técnicas }\end{array}$ & $\begin{array}{l}\text { 1. Conocimiento, formación, preparación y } \\
\text { competencia profesional. } \\
\text { 2. Formación continua } \\
\text { 3. Innovación y superación } \\
\text { 4. Competencia profesional (técnica) }\end{array}$ \\
\hline Competencias sociales & $\begin{array}{l}\text { 5. Compañerismo y relaciones } \\
\text { 6. Comunicación } \\
\text { 7. Trabajar en equipo } \\
\text { 8. Ser trabajador }\end{array}$ \\
\hline Competencias éticas & $\begin{array}{l}\text { 9. Responsabilidad } \\
\text { 10. Honestidad } \\
\text { 11. Ética profesional y personal } \\
\text { 12. Prestar mejor servicio a la sociedad } \\
\text { 13. Respeto } \\
\text { 14. Principios morales y valores profesionales }\end{array}$ \\
\hline $\begin{array}{l}\text { Competencias afectivo- } \\
\text { emocionales }\end{array}$ & $\begin{array}{l}\text { 15. Identificarse con la profesión } \\
\text { 16. Capacidad emocional }\end{array}$ \\
\hline
\end{tabular}

Fuente: Hirsch (2005)

\section{Características de la muestra}

La Facultad de Idiomas cuenta con unidades académicas en cuatro municipios del estado de Baja California: Mexicali, Tecate, Tijuana y Ensenada. Los programas de estudios de licenciatura contemplan los primeros dos semestres como tronco común, por lo que los participantes en esta investigación fueron estudiantes que cursaban los semestres de tercero a octavo. Estos últimos integran las etapas disciplinaria (de tercero a sexto) y terminal (séptimo y octavo).

El total de estudiantes en la licenciatura inscritos en las etapas disciplinaria y terminal ascendía a 632 al realizar el estudio, como se muestra en la tabla 2. 
Tabla 2. Población estudiantil de la licenciatura en Docencia de Idiomas

\begin{tabular}{|c|c|c|c|c|c|c|c|c|c|}
\hline \multicolumn{10}{|c|}{ Unidad académica (UA) } \\
\hline \multirow[b]{2}{*}{ Semestre } & \multicolumn{2}{|c|}{ Ensenada } & \multicolumn{2}{|c|}{ Mexicali } & \multicolumn{2}{|c|}{ Tecate } & \multicolumn{2}{|c|}{ Tijuana } & \multirow[b]{2}{*}{ Totales } \\
\hline & Mat & Ves & Mat & Ves & Mat & Ves & Mat & Ves & \\
\hline Tercero & 23 & - & 22 & 29 & 35 & - & 40 & 25 & 174 \\
\hline Cuarto & 7 & - & - & 15 & - & - & - & 30 & 52 \\
\hline Quinto & - & 23 & 24 & 15 & 25 & - & 40 & 20 & 147 \\
\hline Sexto & 16 & - & - & 15 & - & - & - & 30 & 61 \\
\hline Séptimo & - & 17 & 26 & 22 & - & - & 30 & 40 & 135 \\
\hline Octavo & - & 11 & - & 15 & - & - & - & 37 & 63 \\
\hline Totales & 46 & 51 & 72 & 111 & 60 & 0 & 110 & 182 & 632 \\
\hline $\begin{array}{r}\text { Total } \\
\text { UA }\end{array}$ & & 97 & & 183 & & 60 & & 292 & 632 \\
\hline
\end{tabular}

Fuente: Elaboración propia a partir de los datos proporcionados por las coordinaciones

Nota: Mat=matutino. Ves=vespertino.

La muestra de los estudiantes de las etapas disciplinaria y terminal se determinó de forma aleatoria estratificada. La intención fue contar con representatividad de estudiantes en las cuatro unidades académicas, así como en cada semestre y turnos de la carrera.

Se aplicaron cuestionarios a 322 estudiantes, lo que representó $50.95 \%$ de la población de la licenciatura en Docencia de Idiomas. Se determinó $95 \%$ de nivel de confianza y un margen de error de $4 \%$. La muestra quedó integrada por $47.2 \%$ del turno matutino y $52.8 \%$ del turno vespertino; se contó con la participación de $62.8 \%$ del género femenino y $37.2 \%$ del masculino (tabla 3). El sexo femenino es una población predominante en el área de formación para la enseñanza de lenguas; ello coincide con los hallazgos documentados en el campo de conocimiento de la investigación para la enseñanza y aprendizaje de lenguas referidos por Ramírez (2013).

Tabla 3. Participantes por unidad académica, turno y sexo

\begin{tabular}{|l|r|r|r|r|r|r|r|r|r|}
\hline Unidad académica & \multicolumn{4}{|c|}{ Turno } & \multicolumn{4}{|c|}{ Sexo } & N \\
\hline & Mat & $\%$ & Ves & $\%$ & $\mathrm{H}^{*}$ & $\%$ & $\mathrm{M}^{*}$ & $\%$ & Total \\
\hline Mexicali & 39 & 34.2 & 75 & 65.8 & 45 & 39.5 & 69 & 60.5 & 114 \\
\hline Ensenada & 40 & 88.9 & 5 & 11.1 & 11 & 24.4 & 34 & 75.6 & 45 \\
\hline Tecate & 48 & 100 & - & - & 13 & 27.1 & 35 & 72.9 & 48 \\
\hline Tijuana & 25 & 21.7 & 90 & 78.3 & 51 & 44.3 & 64 & 55.7 & 115 \\
\hline TOTAL & 152 & 47.2 & 170 & 52.8 & 120 & 37.2 & 202 & 62.8 & 322 \\
\hline
\end{tabular}

Fuente: Elaboración propia

*Nota: $\mathrm{H}=$ Hombres. $\mathrm{M}=$ Mujeres.

En la tabla 4 se presenta el número de estudiantes participantes de acuerdo con la unidad académica a la que pertenecen y el semestre. 
Tabla 4. Participantes por unidad académica y semestre

\begin{tabular}{|c|c|c|c|c|c|c|c|c|c|c|c|c|c|}
\hline \multirow{2}{*}{$\begin{array}{l}\text { Unidad } \\
\text { académica }\end{array}$} & \multicolumn{12}{|c|}{ Semestre } & \multirow[t]{2}{*}{ Total } \\
\hline & $3 .^{\circ}$ & $\%$ & $4 .^{\circ}$ & $\%$ & $5 .^{\circ}$ & $\%$ & $6^{\circ} .^{\circ}$ & $\%$ & $7 .^{\circ}$ & $\%$ & $8^{\circ}$ & $\%$ & \\
\hline Mexicali & 33 & 28.9 & 11 & 9.6 & 22 & 19.3 & 10 & 8.8 & 24 & 21.1 & 14 & 12.3 & 114 \\
\hline Ensenada & 13 & 28.9 & 22 & 48.9 & - & - & - & - & 5 & 11.1 & 5 & 11.1 & 45 \\
\hline Tecate & 29 & 60.4 & - & 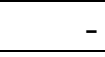 & 19 & 39.6 & & - & - & 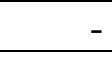 & - & & 48 \\
\hline Tijuana & - & - & 18 & 15.7 & 39 & 33.9 & 19 & 16.5 & 14 & 12.2 & 25 & 21.7 & 115 \\
\hline TOTAL & 75 & 23.29 & 51 & 15.83 & 80 & 24.85 & 29 & 9.0 & 43 & 13.35 & 44 & 13.66 & 322 \\
\hline
\end{tabular}

Fuente: Elaboración propia

\section{Proceso de análisis de datos}

Para identificar los tres rasgos más importantes por unidad académica, semestre, sexo, turno, así como para contrastar por unidad académica, los datos se procesaron en el software estadístico Statistical Package for the Social Sciences (SPSS). En primer lugar, se obtuvieron las frecuencias de los rasgos. Posteriormente, se elaboraron tablas de contingencia para visualizar los datos de forma conjunta y realizar el análisis según las variables ya establecidas.

Para determinar las diferencias significativas en las distintas categorías de los datos, fue necesario, inicialmente, identificar la normalidad de los datos. Posteriormente se llevaron a cabo distintos análisis; para el caso de las variables donde se comparan varios grupos se utilizó el análisis de varianza (Anova), que es el caso de las comparaciones entre unidades académicas y semestres; para aquellas variables donde se comparan solo dos grupos, el análisis fue la prueba T para muestras independientes. Lo anterior asumiendo la normalidad de los datos; en caso contrario, se recurre a las pruebas no paramétricas.

\section{Análisis de resultados}

Se presentan los resultados de la percepción de los alumnos sobre los rasgos de ser un buen profesional, según las categorías de análisis expuestas con anterioridad. Los tres rasgos más importantes por unidad académica se exponen en la tabla 5; luego se muestran los hallazgos de las variables semestre, sexo y turno que sean distintos. Se encontró similitudes en la categorización en todas las variables, por lo que solo se destacarán los que aporten diferencias a lo hallado en la de unidad académica.

A partir de la prueba de normalidad de la distribución de los datos, se encuentra significación estadística ( $\mathrm{p}<0.05)$ en todas las categorías, excepto en el rasgo clasificado como tercero en importancia, en la categoría Ensenada (normalidad de la distribución), lo 

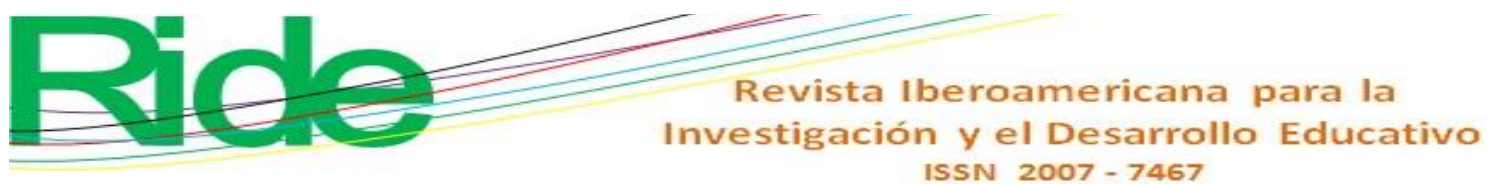

que lleva a asumir la no normalidad en la distribución de la variable unidad académica en la muestra analizada. Se llevaron a cabo análisis no paramétricos para identificar diferencias significativas.

Al tratarse de distribuciones no normales en la muestra analizada, se utilizó la prueba de Kruskal-Wallis, excepto para el rasgo tercero en importancia en Ensenada; para tal caso se utilizó la prueba de Anova.

Se interpreta la significancia asintótica (bilateral), la cual en todos los casos es mayor a .05 , lo cual nos permite concluir que no existe una asociación estadísticamente significativa entre unidad académica y la elección de un rasgo profesional en ninguna de las categorías exploradas.

Ahora, analizando el caso del tercer rasgo en importancia, se llevó a cabo el análisis Anova, con el cual se encontró lo siguiente. A partir de la prueba de homogeneidad de varianzas, con el estadístico de Levene, identificamos que su nivel de significancia es menor a .05 , lo que indica que no existe homegeneidad de varianza en los datos analizados. Al realizar la prueba del estadístico $\mathrm{F}$, su significancia es superior a .05, lo cual apunta a que no existen diferencias significativas entre unidades académicas al momento de elegir el rasgo profesional.

Tabla 5. Rasgos más importantes por unidad académica

\begin{tabular}{|c|c|c|c|c|c|c|c|c|}
\hline \multirow{2}{*}{ Posición } & \multicolumn{8}{|c|}{ Unidad académica } \\
\hline & Ensenada & $\%$ & Mexicali & $\%$ & Tecate & $\%$ & Tijuana & $\%$ \\
\hline $\begin{array}{l}\text { Primero en } \\
\text { importancia }\end{array}$ & $\begin{array}{l}\text { Conocimiento, } \\
\text { formación, } \\
\text { preparación y } \\
\text { competencia } \\
\text { profesional }\end{array}$ & 60 & $\begin{array}{l}\text { Conocimiento, } \\
\text { formación, } \\
\text { preparación y } \\
\text { competencia } \\
\text { profesional }\end{array}$ & 67.5 & $\begin{array}{l}\text { Conocimiento, } \\
\text { formación, } \\
\text { preparación y } \\
\text { competencia } \\
\text { profesional }\end{array}$ & 60.4 & $\begin{array}{l}\text { Conocimiento, } \\
\text { formación, } \\
\text { preparación y } \\
\text { competencia } \\
\text { profesional }\end{array}$ & 69.6 \\
\hline $\begin{array}{l}\text { Segundo en } \\
\text { importancia }\end{array}$ & $\begin{array}{l}\text { Ética } \\
\text { profesional y } \\
\text { personal }\end{array}$ & 20 & $\begin{array}{l}\text { Ética } \\
\text { profesional y } \\
\text { personal }\end{array}$ & 18.4 & $\begin{array}{l}\text { Conocimiento, } \\
\text { formación, } \\
\text { preparación y } \\
\text { competencia } \\
\text { profesional }\end{array}$ & 27.1 & Responsabilidad & 20.2 \\
\hline $\begin{array}{l}\text { Tercero en } \\
\text { importancia }\end{array}$ & $\begin{array}{l}\text { Formación } \\
\text { continua }\end{array}$ & 15.6 & $\begin{array}{l}\text { Ética } \\
\text { profesional y } \\
\text { personal }\end{array}$ & 15.8 & $\begin{array}{l}\text { Ética } \\
\text { profesional y } \\
\text { personal }\end{array}$ & 29.2 & $\begin{array}{l}\text { Innovación y } \\
\text { superación }\end{array}$ & 17.5 \\
\hline
\end{tabular}

Fuente: Elaboración propia

De forma general (tabla 5), se identifica que el rasgo más valorado se ubica en las competencias cognitivas y técnicas, pues se sitúa el conocimiento, formación, preparación y competencia profesional como el más importante; en segundo y tercer lugar, con el mismo número de frecuencias, la ética profesional y personal, rasgo que corresponde a las 


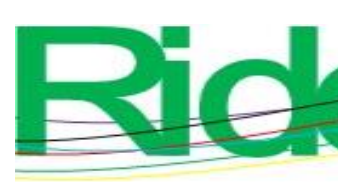

Revista Iberoamericana para la Investigación y el Desarrollo Educativo ISSN 2007 - 7467

competencias éticas. En Ensenada y Tijuana, se encuentran dos rasgos categorizados que pertenecen a las competencias cognitivas y técnicas (formación continua e innovación y superación), mientras que en esta última unidad académica ubican en segundo lugar a un rasgo de las competencias éticas (responsabilidad). Entre los rasgos categorizados con el menor número de menciones se encuentra capacidad emocional, de las competencias afectivo-emocionales. En la unidad académica de Ensenada se hace la mención del rasgo prestar mejor servicio a la sociedad, de las competencias sociales, en último lugar.

En la variable semestre se encontraron similares resultados. Al tratarse de distribuciones no normales en la muestra analizada, se llevó a cabo la prueba de KruskalWallis. La significancia asintótica en todos los casos es mayor a .05, lo cual nos permite concluir que no existe una asociación estadísticamente significativa entre semestre y la elección de un rasgo profesional en ninguna de las categorías exploradas.

Los resultados de los tres rasgos más valorados por semestre coinciden con los encontrados en la variable unidad académica, donde de acuerdo con la clasificación por competencias se valora el rasgo conocimiento, formación, preparación y competencia profesional en los primeros lugares. Se encontró que en quinto y sexto semestre emergen valores como innovación y superación (15\%), así como formación continua (13.8 \%), respectivamente, los cuales corresponden a las competencias cognitivas y técnicas; además principios morales y valores profesionales, de las competencias éticas, en tercero (17.3\%) y octavo semestre (20.8\%). No obstante, prevalecen los rasgos capacidad emocional y prestar mejor servicio a la sociedad entre los menos importantes.

En las variables sexo y turno, se realizaron pruebas no paramétricas, en este caso, la U de Mann-Whitney, apropiada para variables con dos categorías, cuya distribución es no normal, al encontrarse una significación estadística de $\mathrm{p}<0.05$. Se concluyó que no existe una asociación estadísticamente significativa entre estas variables y en la elección de un rasgo profesional en ninguna de las categorías exploradas.

Los resultados no varían de lo ya planteado, a excepción de la categoría de sexo femenino, donde emerge el valor responsabilidad como segundo en importancia con $17.4 \%$, mismo que corresponde a las competencias éticas. En tercer lugar, ambos sexos ubican a la ética profesional y personal (mujeres con $15.3 \%$ y hombres con $16.8 \%$ ).

En la variable turno prevalecen los rasgos más valorados que se han mencionado con antelación. Se encontró en ambos turnos que los valores conocimiento, formación, preparación y competencia profesional (matutino en primero [63.2\%] y segundo lugar [16.4 


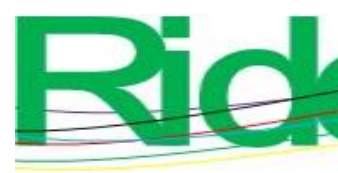

Revista Iberoamericana para la Investigación y el Desarrollo Educativo ISSN 2007 - 7467

$\%$ ]; vespertino en primer lugar [68.8\%]), así como ética profesional y personal (matutino en tercer lugar [16.4\%] y vespertino en segundo [16.6\%]) son los de más alta frecuencia. En el turno vespertino, se encuentra como tercero en importancia el valor principios morales y profesionales con $16.6 \%$.

En los menos valorados de las variables sexo y turno no se hallaron diferencias con lo anteriormente planteado en la variable unidad académica.

\section{Discusión}

En los rasgos analizados se encuentran resultados similares entre las categorías presentadas (por unidad académica, semestre, sexo y turno). Los profesores de idiomas en formación destacan entre los rasgos profesionales más importantes el conocimiento, formación, preparación y competencia profesional y ética profesional. Según lo planteado, la formación profesional consiste en adquirir los conocimientos de la disciplina; para ser parte de la profesión se accede "a ella tras un largo proceso de capacitación teórica y práctica, de la cual depende la acreditación o licencia para ejercer dicha profesión” (Hortal, 2002, p. 51), y de ello parecen ser conscientes los profesores de idiomas.

Es importante resaltar que los rasgos mencionados entre los tres más importantes, con distintas frecuencias, pertenecen a las competencias cognitivas y técnicas: 1) conocimiento, formación, preparación y competencia profesional, 2) innovación y superación y 3) formación continua. Estos rasgos de los valores profesionales permiten aseverar que los estudiantes identifican la adquisición de conocimientos como determinantes para desempeñarse en su profesión, lo cual es congruente al tener la intención de acceder a estudios universitarios, ya que los propósitos de estas instituciones son formar a sus estudiantes con las capacidades para insertarse en un mundo globalizado, aunque — vale resaltar - sin olvidar la responsabilidad social.

Las instituciones de educación superior tienen entre sus funciones sustantivas tres puntos rectores que guían sus acciones: "La gestión socialmente responsable de la formación, la gestión socialmente responsable del conocimiento y la cultura, y la gestión socialmente responsable de la institución en su conjunto", también denominado como "campus responsable" (Anuies, 2012, en UABC, 2017, p. 8). Se puede afirmar que los profesores de idiomas en formación, sea por el discurso colectivo en el que están inmersos — en la casa, en la escuela, en la comunidad-, conciben como primordiales los valores relacionados con la 


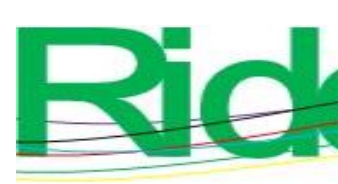

Revista Iberoamericana para la Investigación y el Desarrollo Educativo ISSN 2007 - 7467

competencia cognitiva y técnica. Esto es lo que les permitirá identificarse como expertos competentes en su profesión, al contar con especialización en el área en que se desempeñarán y obtener la acreditación académica.

Por otra parte, no se observa que identifiquen otros rasgos como los categorizados en las competencias sociales y afectivo-emocionales. Ello a pesar de que en el plan de estudios se da énfasis al desarrollo de habilidades para trabajar en equipo, de comunicación, de establecer relaciones de compañerismo, así como para cumplir de forma óptima con las tareas asignadas. La escasa valorización a estos rasgos coincide con los hallazgos de Luna-Serrano et al. (2010). Además, se podría indagar en si el exiguo valor que atribuyen a las competencias sociales tiene relación con la baja apreciación del profesorado respecto a esta dimensión — como fue identificado en el trabajo de Serna y Luna (2011)—, por lo que no se desarrolla en la formación profesional de los sujetos estudiados. También habría que analizar por qué ubican a la capacidad emocional entre los menos importantes, cuando en el desempeño docente esta capacidad coadyuva a establecer un clima propicio para el aprendizaje. En este sentido, vale destacar que a través de la identificación de sus propios sentimientos y los de los demás se pueden establecer relaciones cordiales y manejar situaciones conflictivas que puedan surgir en el aula o en su ambiente de trabajo.

El rasgo identificarse con la profesión, parte de la identidad profesional, es un elemento esencial que se debe desarrollar en el currículo del programa educativo, ya que hasta ahora es un aspecto que no se identifica como preponderante entre los estudiantes. Es el desarrollo del ethos profesional, mismo que en palabras de López Zavala (2013) "toma sentido pleno no en escenarios imaginados sino en ámbitos socialmente existentes, lo cual contribuye al desarrollo de la profesionalidad moral (p. 18)", donde — como ya se expresósí es preponderante el conocimiento teórico y práctico para su desempeño en el campo disciplinar, aunque la vocación y la apropiación de las normas de conducta de su profesión son fundamentales, ya que entonces sus acciones tendrán "como divisa la pretensión de justicia en la relación con su comunidad” (López Zavala, 2013, p. 18).

En México, en el campo de la enseñanza de lenguas, la identidad del profesor se encuentra fragmentada debido a diversos factores. Primordialmente, a los vacíos en la inserción de profesores — con formación universitaria en el área de la enseñanza de lenguas - en los espacios educativos. Esta situación es generada por la valoración que dan a la competencia comunicativa en la lengua de instrucción como el único factor a considerar 


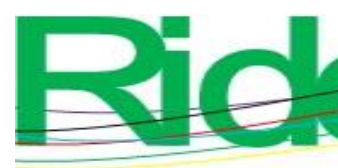

Revista Iberoamericana para la
Investigación y el Desarrollo Educativo
ISSN $2007-7467$

para la contratación. Estas circunstancias afectan la construcción de la identidad profesional y las representaciones que tienen los sujetos acerca del sentido de ser docente.

Las formas de comportamiento que se van construyendo a partir de la práctica y discurso de un gremio profesional van aportando hacia la interpretación aceptada sobre las conductas que son apropiadas y categorizadas como correctas desde una ética profesional. Son las que generan valores y patrones de conducta legitimizados que se trasmiten como tradición en el ejercicio profesional (López Calva, 2009). Por ello, "los principios son imperativos de tipo general, que orientan acerca de lo que es bueno hacer y lo que debe evitarse. Señalan grandes temas y valores de referencia, hay que tener en cuenta a la hora de decidir y de enfrentar casos problemáticos" (Hirsch, 2013, p. 105).

Esta incidencia en los resultados puede interpretarse como que los estudiantes no alcanzan a discriminar entre los rasgos, por lo que eligen los que les son más familiares, aunque ello resulte en discordancias. Aquí habría que cuestionarse cómo se abordan en el desarrollo del currículo por todos los actores que intervienen en la formación que reciben en la institución, dentro y fuera del aula. Este es un elemento que se debe considerar en posteriores investigaciones para indagar respecto a las percepciones, principalmente, de los profesores formadores en el programa educativo y otros sujetos, también con injerencia en él.

Para lograr un acercamiento real sobre el significado de los rasgos de un buen profesional, es necesario indagar en los significados de la práctica que atribuyen los profesores de idiomas en formación. Por ello, el profundizar de manera cualitativa es una alternativa para explicar las diferencias e identificar la formación que reciben respecto a las dimensiones exploradas.

En otras palabras, se deben abordar los significados desde una perspectiva humanística con la finalidad de comprenderlos e interpretarlos, es decir, un enfoque cualitativo (Taylor y Bogdan, 2006) permitirá aclarar la contradicción. De esta forma, mediante métodos interpretativos se podrá explicar lo que sucede en determinado grupo o persona, e identificar la perspectiva de los actores en su contexto para conocer lo que acontece en un lugar particular y los significados que les dan.

Las cuestiones que se identifican para explorar —es decir, examinar las opiniones de otros sujetos relacionados con la formación que reciben los futuros docentes de lenguas, así como utilizar estrategias cualitativas para ahondar en la percepción de los estudiantesrepresentan áreas de oportunidad para futuros estudios del tema abordado en este trabajo. 


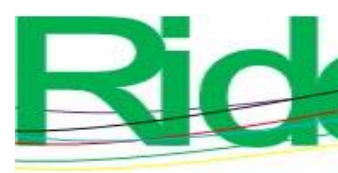

Revista Iberoamericana para la Investigación y el Desarrollo Educativo ISSN $2007-7467$

\section{Conclusiones}

Este estudio tuvo como objetivo identificar los rasgos más importantes desde la opinión de los estudiantes de licenciatura para la enseñanza de idiomas. Esto permitió tener un acercamiento para reconocer tensiones e inconsistencias en la formación inicial de los sujetos respecto a los rasgos de un buen profesional y determinar si constituyen un vacío en el currículo del programa educativo. En el análisis de las variables no se observaron diferencias significativas en las categorías analizadas de unidad académica, semestre, turno o sexo.

Por tanto, la primera conclusión general que se deriva de los resultados es el alto valor que los profesores de lenguas en formación atribuyen a las competencias cognitivas y técnicas, aunque se observan ausencias en las competencias sociales y afectivo-emocionales al no identificar otros rasgos también relevantes para su formación profesional.

Esto permite dilucidar cómo van conformando las representaciones de su profesión, su ethos profesional. Los estudiantes otorgan una alta valoración a los rasgos asociados con la formación y actualización, lo que no se pretende desestimar, ya que son la base del ejercicio docente, el marco de referencia para la planeación y la toma de decisiones. No obstante, aunque la ética profesional y personal es un rasgo con una alta frecuencia, la cual forma parte de las competencias éticas, existe una ausencia en cuanto al compromiso con la sociedad, el respeto y la honestidad, elementos necesarios para conformar un entorno social más justo.

El que la percepción de los estudiantes carezca de los rasgos antes mencionados puede tener impacto en las acciones que realicen en la comunidad, en su entorno social y, por ende, en su ejercicio profesional. En este sentido, la formación implica adquirir habilidades y valores, y no solo conocimientos teóricos y técnicos para autorregularse y guiarse en las formas de relación, y para conducirse con otros sujetos y diversos ambientes.

Los hallazgos aportan datos sobre la relevancia de conformar un lenguaje común donde se incorporen rasgos de las competencias sociales y afectivo-emocionales, así como fortalecer la dimensión ética en el trayecto formativo del programa educativo. Esto de forma explícita para que, mediante la reflexión, los estudiantes puedan analizar y consolidar aspectos que les permitan una formación humanística y de responsabilidad social para impactar de forma favorable en su desempeño profesional. 


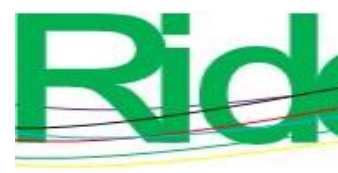

Revista Iberoamericana para la Investigación y el Desarrollo Educativo ISSN $2007-7467$

El análisis de estas dimensiones orienta en las directrices para realizar adecuaciones en la formación de profesores de lenguas. Asimismo, se deben examinar las opiniones de los profesores formadores de estos estudiantes y la forma en que implementan el currículo para determinar si lo realizan de acuerdo con lo que la formación integral precisa, de forma transversal y con un aprendizaje para toda la vida.

Por último, se reflexiona ante el hecho de que los profesores de lenguas se encuentran en una etapa de construcción de la identidad profesional, derivada de las circunstancias que prevalecen en su ámbito educativo y la manera en que se les percibe. Esta es una cuestión que se debe seguir edificando para que sea sólida y se formen las representaciones de ser docente. Aunado a ello, los cambios y transformaciones que se suscitan en las instituciones de educación superior exigen que la formación de profesores de lenguas también se oriente hacia la investigación. Esto ante un mayor número de posgrados en el área de lingüística aplicada, por lo que le da una nueva mirada a la profesión, agregando nuevos espacios profesionales, además de la docencia. Coyuntura que tiene impacto en el ethos profesional y sus actuaciones.

\section{Contribuciones a futuras líneas de investigación}

Como futuras líneas de investigación, se considera relevante profundizar en las opiniones de los estudiantes respecto a los rasgos que categorizan con la más baja valoración, que corresponden a las competencias sociales y afectivo-emocionales. Mediante una metodología cualitativa, será posible identificar su perspectiva. De la misma forma, es necesario indagar las opiniones de otros agentes que intervienen en la formación de los profesores de lenguas y analizar cómo perciben (y viven en el aula) los rasgos de un buen profesional. Los docentes que participan en este proceso formativo son los mediadores en la implementación del modelo curricular, por tanto, tienen gran influencia en el desarrollo del estudiante como parte de su formación profesional. 


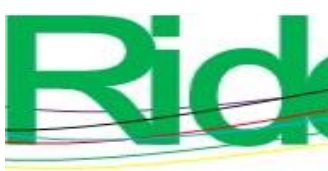

Revista Iberoamericana para la
Investigación y el Desarrollo Educativo
ISSN $2007-7467$

\section{Referencias}

Asociación Nacional de Universidades e Instituciones de Educación Superior [Anuies] (2019). Elementos básicos para el diseño de un programa de formación en enseñanza del inglés. México: Anuies.

Bauman, Z. (2011). La sociedad sitiada. México: Fondo de Cultura Económica.

Beltrán, F., Torres, I., Beltrán, A. y García. F. (2005). Un estudio comparativo sobre valores éticos en estudiantes universitarios. Enseñanza e Investigación en Psicología, 10(2), 397-415.

Bolívar, A. (2007). La formación inicial del profesorado de secundaria y su identidad profesional. Estudios sobre Educación, 12, 13-30. Recuperado de http://dspace.si.unav.es/dspace/bitstream/10171/8987/1/12\%20Estudios\%20Ea.pdf

Breen, M. P. (1991). Understanding the language teacher. In Phillipson, R., Kellerman, E., Selinker, L., Sharwood Smith, M. and Swain, M. (eds.), Foreign/Second Language Pedagogy Research. Clevedon, UK: Multilingual Matters.

Fierro, L. (2019). Retos y oportunidades del Programa Nacional de Inglés. Perspectivas de profesores de primarias públicas. México: UABC.

Fierro, L., Martínez, L. y Cordero, G. (2014). La formación del profesor de inglés en las escuelas primarias públicas. Un caso en Baja California.Educ@UPN, (16).

Freeman, D. (1989). Teacher training, development, and decision making: a model of teaching and related strategies for language teacher education. TESOL Quarterly, 23(1), 27-46.

Hirsch, A. (2005). Construcción de una escala de actitudes sobre ética profesional. Revista Electrónica de Investigación Educativa, 7(1). Recuperado de http://redie.uabc.mx/vol7no1/contenido-hirsch.html

Hirsch, A. (2013). La ética profesional basada en principios y su relación con la docencia. Edetania, (43), 97-111. Recuperado de https://dialnet.unirioja.es/servlet/articulo?codigo $=4406374$

Hirsch, A. y Pérez-Castro, J. (2007). Estado de conocimiento sobre valores profesionales y ética profesional. Recuperado de http://www.reduval.org.mx/files/memoria_ixjornada/files/panel_estados_del_conoci miento/estado_del_conocimiento_sobre_valores_profesionales_y_etica_profesional. pdf 


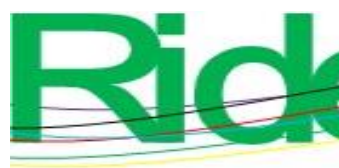

Revista Iberoamericana para la Investigación y el Desarrollo Educativo ISSN $2007-7467$

Hortal, A. (2000). Docencia. En Cortina, A. y Conill, J. (coords.), 10 palabras clave en ética de las profesiones. Navarra: Verbo Divino.

Hortal, A. (2002). Ética general de las profesiones. Bilbao, España: Desclée De Brouwer.

Imbernón, F. (2001). La profesión docente ante los desafíos del presente y del futuro. En Marcelo, C. (ed.), La función docente. España: Síntesis.

Kelly, M., Grenfell, M., Allan, R., Kriza, C. and McEvoy, W. (2004). European Profile of Language Teacher Education - a Frame of Reference. Southampton, UK: University of Southampton.

Lopes, A. (2007). La construcción de identidades docentes como constructo de estructura y dinámica sistémicas: argumentación y virtualidades teóricas y prácticas. Profesorado. Revista de Currículum y Formación del Profesorado, 11(3). Recuperado de http://www.ugr.es/es/local/recfpro/rev113COL1.pdf

López Calva, M. (2009). Hacia una práctica profesional que contribuya a la humanización. Revista Mexicana de Investigación Educativa, 14(43), 1313-1321. Recuperado de http://www.scielo.org.mx/scielo.php?script=sci_arttext\&pid=S140566662009000400015

López Zavala, R. (2013). Ética profesional en la formación universitaria. Perfiles Educativos, $35(142)$.

Luna-Serrano, E., Valle-Espinoza, M. y Osuna-Lever, C. (2010). Los rasgos de un "buen profesional", según la opinión de estudiantes universitarios en México. Revista Electrónica de Investigación Educativa, (número especial). Recuperado de http://redie.uabc.mx/NumEsp2/contenido-luna3.html

Paquay, L., Altet, M., Charlier, E. y Perrenoud, P. (coords.) (2005). La formación profesional del maestro. Estrategias y competencias. México: Fondo de Cultura Económica.

Ramírez, J. L. (coord.). (2007). Las investigaciones sobre la enseñanza y el aprendizaje de lenguas extranjeras en México. México: Plaza y Valdés.

Ramírez, J. L. (coord.). (2010). Las investigaciones sobre la enseñanza y el aprendizaje de lenguas extranjeras en México. Una segunda mirada. México: Cengage Learning.

Ramírez, J. L. (coord.). (2013). Una década de búsqueda: las investigaciones sobre la enseñanza y el aprendizaje de lenguas extranjeras en México (2000-2011). México: Pearson.

Richards, J. C. (1998). Beyond Training. USA: CUP.

Richards, J. C. (2011). Competence and Performance in Language Teaching. USA: CUP. 


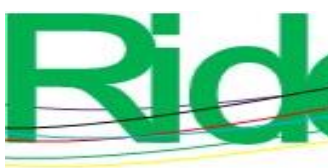

Revista Iberoamericana para la
Investigación y el Desarrollo Educativo
ISSN $2007-7467$

Sanz, R. y Hirsch, A. (2016). Ética profesional en el profesorado de educación secundaria de la Comunidad Valenciana. Perfiles Educativos, 38(151), 139-156. Recuperado de http://www.scielo.org.mx/scielo.php?script=sci_abstract\&pid=S018526982016000100139\&lng=es\&nrm=iso

Schön, D.A. (1983). The Reflective Practitioner. How Professionals Think in Action. USA: Basic Books, Inc.

Secretaría de Educación Pública [SEP] (2011). Acuerdo número 592. Por el que se establece la articulación de la Educación Básica. México: SEP. Recuperado de http://www.reformapreescolar.sep.gob.mx/normatividad/acuerdos/acuerdo_592.pdf

Secretaría de Educación Pública [SEP] (2017). Estrategia nacional de inglés. Comunicado núm. 184. Ciudad de México: SEP. Recuperado de https://www.gob.mx/sep/prensa/comunicado-184-presenta-nuno-mayer-laestrategia-nacional-de-ingles-para-que-mexico-sea-bilingue-en-20-anos

Serna, A. y Luna, E. (2011). Valores y competencias para el ejercicio de la docencia de posgrado. Sinéctica, (37). Recuperado de: http://www.scielo.org.mx/scielo.php?script=sci_arttext\&pid=S1665109X2011000200007\&lng=es\&nrm=iso

Taylor, S. J. y Bogdan, R. (2006). Introducción a los métodos cualitativos de investigación. La búsqueda de significados. Buenos Aires, Barcelona, México: PAIDOS.

Universidad Autónoma de Baja California [UABC] (2014). Modelo educativo de la UABC. Mexicali: UABC.

Universidad Autónoma de Baja California [UABC] (2017). Diagnóstico de la formación de valores y responsabilidad social universitaria en la Universidad Autónoma de Baja California. Mexicali: UABC.

Woods, D. (1996). Teacher Cognition in Language Teaching. Cambridge: CUP. 


\begin{tabular}{|c|c|}
\hline 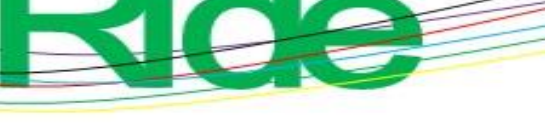 & $\begin{array}{l}\text { Revista lberoamericana para la } \\
\text { Investigación y el Desarrollo Educativo } \\
\text { ISSN } 2007-7467\end{array}$ \\
\hline Rol de Contribución & Autor (es) \\
\hline Conceptualización & Laura Emilia Fierro López \\
\hline Metodología & $\begin{array}{l}\text { Laura Emilia Fierro López (principal), Rey David Román } \\
\text { Gálvez (que apoya) }\end{array}$ \\
\hline Software & Rey David Román Gálvez \\
\hline Validación & Laura Emilia Fierro López \\
\hline Análisis Formal & $\begin{array}{l}\text { Laura Emilia Fierro López (que apoya), Rey David Román } \\
\text { Gálvez (principal) }\end{array}$ \\
\hline Investigación & Laura Emilia Fierro López \\
\hline Recursos & Laura Emilia Fierro López \\
\hline Curación de datos & $\begin{array}{l}\text { Laura Emilia Fierro López (principal), Rey David Román } \\
\text { Gálvez (que apoya), Lilia Martínez Lobatos (que apoya). }\end{array}$ \\
\hline $\begin{array}{l}\text { Escritura - Preparación del } \\
\text { borrador original }\end{array}$ & Laura Emilia Fierro López \\
\hline Escritura - Revisión y edición & $\begin{array}{l}\text { Laura Emilia Fierro López (principal), Rey David Román } \\
\text { Gálvez (que apoya), Lilia Martínez Lobatos (que apoya). }\end{array}$ \\
\hline Visualización & Laura Emilia Fierro López \\
\hline Supervisión & Laura Emilia Fierro López \\
\hline Administración de Proyectos & Laura Emilia Fierro López \\
\hline Adquisición de fondos & Laura Emilia Fierro López \\
\hline
\end{tabular}

